\title{
Utilização de Plantas Medicinais com Atividade Antimicrobiana por Usuários do Serviço Público de Saúde em Campina Grande - Paraíba
}

SOUZA, C.M.P1; BRANDÃO, D.O.'; SILVA, M.S.P.2; PALMEIRA, A.C. ${ }^{3}$; SIMÕES, M.O.S. ${ }^{1,3}$; MEDEIROS, A.C.D..$^{12^{*}}$ ${ }^{1}$ Departamento de Farmácia, Centro de Ciências Biológicas e da Saúde, Universidade Estadual da Paraíba. Rua Baraúnas, 351, Campus I, Bodocongó, Campina Grande, Paraíba, Brasil. CEP: 58.429 - 500. *E-mail: anacdmedeiros@yahoo.com.br. ${ }^{2}$ Mestrado em Odontologia, Centro de Ciências Biológicas e da Saúde, Universidade Estadual da Paraíba. Campina Grande, Paraíba, Brasil. CEP: 58.429 - 500. ${ }^{3}$ Mestrado em Saúde Pública, Centro de Ciências Biológicas e da Saúde, Universidade Estadual da Paraíba. Campina Grande, Paraíba, Brasil. CEP: 58.429 - 500.

RESUMO: O estudo avalia a utilização de plantas medicinais com atividade antimicrobiana pelos usuários do Sistema Único de Saúde do município de Campina Grande- PB, Brasil. Trata-se de um estudo descritivo, transversal, com abordagem quantitativa, cuja amostra constituiu-se de 220 usuários conduzido no período de Agosto de 2008 a Janeiro de 2009. A pesquisa foi realizada através de um roteiro de entrevistas não estruturada. Dentre os participantes do estudo, $65,0 \%$ utilizavam plantas medicinais, das quais Punica granatum L., Anacardium occidentale L., e Stryphnodendron adstringens foram as mais citadas. Observou-se que houve prevalência do gênero feminino na utilização. A maioria dos indivíduos obtém plantas medicinais no comércio local utilizando-as por indicação de familiares. Foi observado que $5,0 \%$ dos entrevistados afirmam já ter sofrido algum evento adverso decorrente do uso de plantas medicinais. Assim sugere-se que as informações sobre o uso da flora medicinal adquiridas nas comunidades locais, combinadas a estudos químicos/ farmacológicos realizados em laboratórios especializados e a capacitação da equipe de saúde favorecerá a implementação da Portaria n 971/2006, que tem como objetivo a garantia de acesso a plantas medicinais e fitoterápicos com segurança, eficácia e qualidade.

Palavras-chave: Plantas Medicinais, Etnofarmacologia, Saúde Pública.

ABSTRACT: Use of medicinal plants with antimicrobial activity by users of the Public Health System in Campina Grande - Paraíba, Brazil. This study evaluates the use of medicinal plants with antimicrobial activity by users of the Unified Health System in Campina Grande Municipality, Paraíba, Brazil. This is a descriptive and transversal study with a quantitative approach, the sample of which consisted of 220 users, and was carried out from August 2008 to April 2009. The research was developed through an unstructured interview process. Among the study participants, $65.0 \%$ used medicinal plants, of which Punica granatum L., Anacardium occidentale L. and Stryphnodendron adstringens were most cited. There was prevalence of females. Most individuals obtain medicinal plants in the local market, using them as suggested by relatives. Results indicated that $5.0 \%$ of the interviewees stated to have suffered adverse event resulting from the use of medicinal plants. Thus, information about the use of medicinal plants, acquired in local communities, combined to chemical/pharmacological studies performed in specialized laboratories and the qualification of health professionals, will favor the implementation of Decree no. 971/2006, which aims to ensure access to medicinal plants and herbal medicines with safety, efficacy and quality.

Key words: Medicinal plants, Ethnopharmacology, Public Health

\section{INTRODUÇÃO}

Apesar das plantas medicinais já fazerem parte da cultura popular, nas últimas décadas o interesse pela Fitoterapia teve um aumento considerável entre usuários, pesquisadores e serviços de saúde. Segundo a Organização Mundial da Saúde (OMS) $80 \%$ da população dos países em desenvolvimento utilizam práticas tradicionais nos seus cuidados básicos de saúde e $85 \%$ usam

Recebido para publicação em 31/07/2011

Aceito para publicação em 26/04/2012

Rev. Bras. PI. Med., Campinas, v.15, n.2, p.188-193, 2013. 
plantas medicinais ou preparações destas. Desde então, a OMS tem expressado a sua posição a respeito da necessidade de valorizar a utilização de plantas medicinais no âmbito sanitário e na atenção básica à saúde (Rosa et al., 2011).

O sistema público de saúde no Brasil não possui uma política de assistência farmacêutica capaz de suprir as necessidades medicamentosas da população, sobretudo no nordeste brasileiro, no qual a população carente apresenta dificuldades para obter os medicamentos essenciais. Alguns estados e municípios brasileiros vêm realizando nas duas últimas décadas a implantação de Programas de Fitoterapia na atenção primária, com o intuito de suprir as carências medicamentosas de suas comunidades. E muitos desses programas estão sendo desenvolvidos no sistema de saúde vinculados ao Programa Saúde da Família (PSF) (Silva et al., 2006).

Segundo Brasileiro et al. (2008), o estudo de plantas medicinais, a partir de seu emprego pelas comunidades, pode fornecer informações úteis para a elaboração de estudos farmacológicos, fitoquímicos e agronômicos sobre estas plantas, com grande economia de tempo e dinheiro. Desta forma, podemos planejar a pesquisa a partir de conhecimento empírico já existente, muitas vezes consagrado pelo uso contínuo, que deverá ser testado em bases científicas.

Para Marinho et al. (2011), os estudos relacionados com a medicina popular têm merecido cada vez mais atenção, em virtude da gama de informações e esclarecimento à ciência. Para o estado da Paraíba, os estudos etnobotânicos ainda são escassos, mas estão gradativamente sendo realizados, com a finalidade de se conhecer as plantas mais utilizadas por esta população.

Plantas nativas da região semiárida têm sido usadas durante séculos, através da extração seletiva de algumas espécies e a utilização de extensas áreas de terra para pastagem ou para a agricultura. A vegetação semiárida do nordeste do Brasil foi reduzida para menos de $50 \%$ de sua cobertura original. Em alguns estados, como o da Paraíba, apenas 33\% da vegetação nativa permanece. Algumas populações no Nordeste do Brasil são muito pobres e dependem muito do uso direto dos recursos naturais para sua subsistência. E a utilização de produtos, derivados da vegetação nativa, especialmente de plantas medicinais, contribui significativamente para a manutenção da qualidade de vida dessa população (Lucena et al., 2007).

As dificuldades encontradas na utilização de plantas medicinais e no desenvolvimento de fitoterápicos são, por exemplo, os problemas econômicos, a inexistência de estudos organizados e integrados e até pouco tempo atrás a ausência de uma política governamental. No Brasil, esse tema vem sendo discutido há alguns anos e a partir dessas discussões foram criadas várias resoluções expressando a necessidade de valorizar o uso desses medicamentos. Dentre elas, a Portaria $n^{\circ}$ 971/2006, que aprova a Política Nacional de Práticas Integrativas e Complementares (PNPIC). Essa portaria tem como objetivo a ampliação das opções terapêuticas aos usuários do Sistema Único de Saúde (SUS) com garantia de acesso a plantas medicinais, medicamentos fitoterápicos, sempre voltados à segurança, eficácia, qualidade e integralidade da atenção à saúde de todos os brasileiros. As Diretrizes para Plantas Medicinais e Fitoterapia afirmam que deve haver uma Relação Nacional de Plantas Medicinais e Fitoterápicos, que contenha lista de espécies com informações sobre seus usos por comunidades locais (Brasil, 2006a; BRASIL 2006b).

Desta forma objetivou-se nesse estudo investigar a utilização de plantas medicinais, com atividade antimicrobiana, pela população usuária do Sistema Único de Saúde, do município de Campina Grande - PB, com a finalidade de se conhecer as plantas mais utilizadas por esta população, o que favorecerá os profissionais de saúde na elaboração de uma possível relação municipal de plantas medicinais, com eficácia, segurança e qualidade.

\section{MATERIAL E MÉTODO}

A cidade de Campina Grande está localizada no Agreste Paraibano, distante $120 \mathrm{~km}$ de João Pessoa, capital do Estado da Paraíba. Segundo dados do Instituto Brasileiro de Geografia Estatística (IBGE 2010) possuem uma área territorial de 621 $\mathrm{Km}^{2}$ e uma população de 383.941 habitantes, sendo que $41,7 \%$ são homens e $55,8 \%$ são mulheres. Como sua posição geográfica é privilegiada, Campina Grande torna-se um pólo de convergência com aproximadamente 232 municípios, não só da Paraíba, como também de estados vizinhos, cujos habitantes se deslocam em busca dos serviços oferecidos, entre os quais os de saúde.

A pesquisa foi realizada em cinco centros de saúde, que atende a população usuária do SUS, do município de Campina Grande, localizados nos bairros: Centro, Bela Vista, Liberdade, Palmeira e Catolé.

A população foi constituída por 220 indivíduos de ambos os sexos, maiores de 18 anos, assistidos pelos centros de saúde acima citados. Tratou-se de um estudo transversal, descritivo, participativo e exploratório, seguido de análise experimental, com abordagem quantitativa.

A pesquisa foi realizada durante o período

Rev. Bras. PI. Med., Campinas, v.15, n.2, p.188-193, 2013. 
de agosto de 2008 a janeiro de 2009. Utilizou-se como instrumento de coleta de dados um formulário que foi preenchido através de dados coletados pelos pacientes em entrevistas não estruturada. Os participantes foram investigados quanto à utilização de plantas medicinais com atividade antimicrobiana, possíveis eventos adversos ocorridos com a utilização de plantas medicinais e quanto ao seu perfil sócio-econômico-demográfico.

Os dados foram submetidos a tratamento estatístico, sendo expostos de maneira descritiva em percentuais simples. Para análise dos dados foi utilizado o Software Epi Info (versão 3.4.3) e Microsoft Office Excel 2007, sendo os mesmos apresentados em figuras e tabelas, considerandose os valores relativos e absolutos que justifiquem a prevalência das respostas.

A pesquisa foi aprovada pelo Comitê de Ética e Pesquisa, da Universidade Estadual da Paraíba, sob protocolo de aprovação $\mathrm{n}^{\circ}$ 0376.0.133.000-08, conforme os padrões exigidos pela Declaração de Helsinque. Os pacientes foram informados antecipadamente sobre os objetivos do estudo, e lhes foram garantidos o direito de desistir da participação na pesquisa, bem como o sigilo das informações colhidas, salvaguardando o direito da privacidade. Houve um termo de consentimento livre e esclarecido, que foi assinado pelo pesquisado e o pesquisador.

\section{RESULTADOS E DISCUSSÃO}

Os resultados mostram que, a maioria da população estudada $95,9 \%$ ( $n=211)$ foi constituída por mulheres. Estes dados estão de acordo com uma pesquisa realizada nas unidades básicas de atenção à saúde da família no município de Maracanaú (CE), na qual $85,4 \%$ dos pacientes entrevistados foram do sexo feminino (Silva et al., 2006). Os resultados concordam também com as pesquisas realizadas sobre a utilização de plantas medicinais em comunidades por Marinho et al., 2011 e Albertasse et al. 2010.

Os resultados deste estudo chamam atenção para a faixa etária prevalente, situada entre 18 e 30 anos de idade $(29,0 \%, n=64)$. Esse dado mostra que a maioria dos pacientes era jovem, diferentemente do que ocorreu em pesquisas etnodirigidas realizadas no estado de Minas Gerais, as quais constataram na maioria de idosos dentre os entrevistados (Brasileiro et al., 2008; Calábria et al., 2008).

Quanto à escolaridade, houve predomínio daqueles que cursaram o ensino fundamental incompleto $(40,9 \%, n=90)$. Este dado reflete as condições educacionais dos entrevistados e está de acordo com levantamentos etnobotânicos e etnofarmacológicos realizados em outras regiões do Brasil (Rossato \& Barbieri, 2007; Silva et al., 2009; Silva et al., 2010).

A renda familiar de $61,4 \%(n=135)$ dos pesquisados situou-se entre 1 e 2 salários mínimos, retratando o baixo poder aquisitivo da população, que não tendo como custear suas necessidades básicas de saúde, busca assistência no SUS. Resultado semelhante foi encontrado em estudo realizado no município de Datas - MG, no qual se verificou que dentre 500 entrevistados $72,0 \%$ tinham baixa renda (Arnous et al., 2005).

Quanto à facilidade de acesso ao serviço de saúde, $68,2 \%(n=150)$ afirmaram que tiveram acesso fácil e $70,0 \%(n=154)$ relatam estar satisfeitos com o serviço de saúde público brasileiro. Esse estudo corroborou com outro realizado na região sul do Brasil, o qual observou que a satisfação comumente está associada à resolutividade do problema e à disponibilidade de medicamentos nos centros de saúde (Halal et al., 1994).

Quanto às características relacionadas à utilização de plantas medicinais, observou-se que a maioria dos indivíduos $88,6 \%$ ( $n=195)$ utiliza plantas medicinais, como alternativa terapêutica para diferentes problemas de saúde, em seu cotidiano. Em pesquisa semelhante, os autores verificaram que $36,5 \% \quad(n=895)$ dos entrevistados utilizam plantas medicinais com freqüência, 55,5\% $(n=1361)$ utilizam raramente e apenas $8,0 \%(n=198)$ não utilizavam (Brasileiro et al., 2008). Essa utilização como opção de tratamento e cura tem aumentado em todas as classes sociais das mais diversas regiões do mundo. A Organização Mundial de Saúde (OMS) estima que entre 65,0 a $80,0 \%$ das pessoas que vivem nos países em desenvolvimento confiam na medicina tradicional para assistência primária da saúde (Gonçalves et al., 2005).

Quanto ao uso de plantas medicinais, especificamente, com atividade antimicrobiana constatou-se que $65,0 \%(n=143)$ dos participantes as utilizam em processos infecciosos. Cada usuário relatou em média duas plantas com atividade antimicrobiana, sendo que para fins de discussão foram consideradas apenas as plantas com freqüência de citação (\%) $\geq 3,0 \%$. Das diferentes plantas medicinais citadas, a prevalência foi da romã (Punica granatum L.), família Lythraceae, com 42,0\% das citações; seguida pelo cajueiro roxo (Anacardium occidentale L.), família Anacardiaceae, com 11,0\%; babatenon (Stryphnodendron coriaceum Benth.), família Fabaceae Lindl., com $6,0 \%$; quixabeira (Bumelia sartorum M.), família Sapotaceae, com 4,0\%; e babosa (Aloe vera L.), família Xanthorrhoeaceae, com 3,0\%. A parte vegetal mais utilizada foi à casca, sendo as plantas adquiridas principalmente no comércio. A forma de 
utilização foi a líquida, sendo preparada através da maceração e administrada por via oral.

Essa pesquisa concorda em parte com a realizada por Marinho et al. (2011), no sertão da Paraíba, que também observou a utilização de cajueiro roxo (Anacardium occidentale L.), babatenon (Stryphnodendron coriaceum Benth.) e quixabeira (Bumelia sartorum M.) por aquela população, sendo também a casca, a parte vegetal mais utilizada e administrada na forma oral. Difere principalmente quanto o local de aquisição das plantas medicinais, que na pesquisa realizada por Marinho et al (2011), 54,0 \% da população consultada as coletavam nas matas.

Foi observado ainda que apenas 5,0\% $(n=11)$ dos pacientes entrevistados relataram a ocorrência de possíveis efeitos adversos após o uso de plantas medicinais. Durante as entrevistas foi registrado algumas frases citadas pela população a respeito da utilização de plantas medicinais, tais como: "é natural, não tem química"; "se bem não fizer, mal não faz"; "tudo sem efeito colateral, não é igual ao da Farmácia"; "não tem contraindicação"; "é bom porque posso tomar quantas vezes eu quiser"; entre outras. De acordo com as informações obtidas, é preocupante o fato de que a maioria dos entrevistados acreditam ser as plantas medicinais destituídas de qualquer efeito adverso, ou até mesmo o fato destas plantas não possuírem contraindicações, já que estudos vêm demonstrando o potencial de toxicidade para várias espécies.

A crença na "naturalidade inócua" dos fitoterápicos e plantas medicinais não é facilmente contradita, pois as evidências científicas de ocorrência de intoxicações e efeitos colaterais relacionados com o uso de plantas medicinais consistem em informações que dificilmente chegam ao alcance dos usuários atendidos nos serviços de saúde pública, caracterizados como indivíduos de baixa escolaridade e acervo cultural (Silveira et al., 2008). A maior parte desses produtos são utilizados por automedicação não tendo o seu perfil tóxico bem conhecido, podendo induzir problemas graves aos usuários, principalmente os mais susceptíveis, como crianças, mulheres grávidas e idosos.

Segundo Marinoff et al. (2009), na Argentina as plantas medicinais quase não são cientificamente validadas e sua segurança e eficácia são freqüentemente desconhecidas e, portanto, como outros medicamentos, devem ser usados com cautela, pois o consumo de plantas tóxicas pode levar a intoxicação e até morte. Casos fatais foram registrados devido ao uso indevido de Chenopodium ambrosioides L., família Amaranthaceae; Alternanthera pungens Kunth, família Amaranthaceae; e Illicium verum Hook. F., família Schisandraceae. Embora possuam propriedades curativas, eles podem deixar as suas condições inócuas por razões diferentes. Mas isso não significa que todos os fitoterápicos sejam prejudiciais; muitas drogas vêm de plantas, e um conhecimento adequado e informações seguras sobre a forma de administração e a posologia são necessárias.

Observou-se ainda que $83,2 \%(n=183)$ dos indivíduos entrevistados obtiveram o conhecimento sobre a forma de utilização de plantas medicinais de familiares, sendo as mães, as principais informantes. Resultado semelhante foi encontrado por Santos et al. (2009) e por Marinho et al. (2011). Esse dado mostra que a medicina popular se fundamenta em um corpo de conhecimento que possui modo de transmissão essencialmente oral e gestual que não se comunica através da instituição médica, mas por intermédio de familiares e vizinhança.

A indicação terapêutica mais frequente, das plantas medicinais utilizadas neste estudo, foi à infecção urinária (Tabela 1).

Em um levantamento etnofarmacológico realizado por Silva et al. (2009), em comunidades rurais do recôncavo baiano observou-se que as plantas mais citadas com atividade antimicrobiana foram: erva-cidreira (Lippia alba Mill.), família Verbenaceae; hortelã grosso (Plectrantus amboinicus (Lour.) Spreng.), família Lamiaceae; e mastruz (Chenopodium ambrosioides L.), família Amaranthaceae, o que deferiu das encontradas neste estudo. As plantas citadas em ambas as

TABELA 1. Correlação entre as planta medicinais com atividade antimicrobiana, sua indicação terapêutica e a forma de utilização.

\begin{tabular}{lcccc}
\hline $\begin{array}{c}\text { Planta } \\
\text { citada }\end{array}$ & $\begin{array}{c}\text { Indicação } \\
\text { terapêutica }\end{array}$ & $\begin{array}{c}\text { Parte da planta } \\
\text { utilizada }\end{array}$ & $\begin{array}{c}\text { Via de } \\
\text { administração }\end{array}$ & $\begin{array}{c}\text { Local de } \\
\text { aquisição }\end{array}$ \\
\hline P. granatum $L$. & Faringite & Casca & Oral & Comércio \\
A. occidentale $L$. & Infecção urinária & Casca & Oral & Comércio \\
S. adstringens $M$. & Infecção urinária & Casca & Oral & Comércio \\
B. sartorum $M$. & Infecção urinária & Casca & Oral & Comércio \\
A. vera $L$. & Furunculose & Folha & Tópico & Domicílio \\
\hline
\end{tabular}

Rev. Bras. PI. Med., Campinas, v.15, n.2, p.188-193, 2013. 
pesquisas já foram bastante mencionadas na literatura científica (Uchgaonkar \& Dahiya, 2011; Al-Zoreky, 2009; Akinpelu, 2001; Pereira et al., 2011; Ruela et al., 2011; Gupta et al., 2010; Hennebelle et al., 2008; Owolabi et al., 2009). Essa perda do conhecimento se deve, entre outros fatores, a sua desvalorização pela sociedade pós-moderna e à crença, pela maioria da população, de que só os medicamentos industrializados são capazes de curar (Almeida et al., 2009; Pilla et al., 2006). No entanto, a Política Nacional de Plantas Medicinais e Fitoterápicos do Brasil tem a intenção de inserir esses produtos no SUS, disponibilizando-os, como alternativa terapêutica, à população usuária deste sistema de saúde, o que certamente contribuirá para que as práticas populares e tradicionais de uso de plantas medicinais e remédios caseiros voltem a ser reconhecidos como forma alternativa e também eficaz de tratamento e prevenção de doenças (Brasil, 2006b).

O fato de não terem sido mencionadas plantas ainda desconhecidas pela comunidade científica, ou pouco estudadas, pode ser indício de que a população pesquisada está, gradativamente, perdendo o conhecimento tradicional repassado pelos seus antepassados. Sendo que, nesse âmbito os estudos etnodirigidos são de grande importância, uma vez que possibilitam o resgate de informações valiosas para a construção do conhecimento científico e o aprimoramento da saúde pública, bem como a descoberta de novas espécies de plantas com ação terapêutica.

Assim, conclui-se que as espécies mais citadas pelos usuários dos centros de saúde do município de Campina Grande/PB com atividade antimicrobiana foram: romã (Punica granatum L.), cajueiro roxo (Anacardium occidentale L.), babatenon (Stryphnodendron coriaceum Benth.), quixabeira (Bumelia sartorum M.) e babosa (Aloe vera L.).

Os resultados deste estudo servem de subsídio para que o conhecimento popular gerado nessa comunidade possa ser compartilhado com as gerações mais jovens, tornando-se necessário o desenvolvimento de estratégias para que essas populações despertem o interesse nessa área, como a criação de espaços, de hortos medicinais ou Farmácias Vivas, onde esse conhecimento possa ser mantido e fortalecido através de mecanismos de geração de renda e de valorização do conhecimento. Combinar as informações adquiridas nas comunidades locais, que fazem uso da flora medicinal, com estudos químicos/farmacológicos realizados em laboratórios especializados tendo como método a formulação de hipóteses referentes às atividades farmacológicas e às substâncias ativas responsáveis pelas ações terapêuticas, trará benefícios à população assistida com segurança, eficácia e otimização do conhecimento tradicional. O que facilitará, pelo menos para a população estudada, a implantação da Portaria nº 971/2006 do Ministério da Saúde do Brasil.

\section{REFERÊNCIAS}

AKINPELU, D. A. Antimicrobial activity of Anacardium occidentale Linn. bark. Fitoterapia, v. 72, p. 287-289, 2001.

ALBERTASSE, P.D.; THOMAZ, L.D.; ANDRADE, M.A. Plantas medicinais e seus usos na comunidade da Barra do Jucu, Vila Velha, ES. Revista Brasileira de Plantas Medicinais, v.12, n.3, p.250-260, 2010.

ALMEIDA, N.F.L; SILVA, S.R.S; SOUZA, J.M; QUEIROZ, A.P.N.; MIRANDA, G.S.; OLIVEIRA, H.B. Levantamento etnobotânico de plantas medicinais na cidade de Viçosa - MG. Revista Brasileira de Farmacognosia, V. 90, N. 4, P. 316-320, 2009.

AL-ZOREKY, N. S. Antimicrobial activity of pomegranate (Punica granatum L.) fruit peels. International Journal of Food Microbiology, v. 134, n. 3, p. 244-248, 2009.

ARNOUS, A.H; SANTOS, A.S; BEINNER, R.P.C. Plantas medicinais de uso caseiro - conhecimento popular e interesse por cultivo comunitário. Revista Espaço para a Saúde, v. 6, n. 2, p. 1-6, 2005.

BRASIL. Ministério da Saúde. A fitoterapia no SUS e - Programa de Pesquisa de Plantas Medicinais da Central de Medicamentos. Brasília: Ministério da Saúde, 148p, 2006a.

BRASIL. Portaria no 971 de 3 de maio de 2006. Aprova a Política Nacional de Práticas Integrativas e Complementares (PNPIC) no SUS. Diário Oficial da República Federativa do Brasil, Brasília, DF, 2006b.

BRASILEIRO, B.G; PIZZIOLO, V.R; MATOS, D.S; GERMANO, A.M; JAMAL, C.M. Plantas medicinais utilizadas pela população atendida no Programa de Saúde da Família, Governador Valadares, MG, Brasil. Revista Brasileira de Ciências Farmacêuticas, v. 44, n. 4, p. 629-636, 2008.

CALÁBRIA, L.; CUBA, G.T.; HWANG, S.M; MARRA, J.C.F.; MENDONÇA, M.F.; NASCIMENTO, R.C. Levantamento etnobotânico e etnofarmacológico de plantas medicinais em Indianópolis, Minas Gerais, Brasil. Revista Brasileira de Plantas Medicinais, v. 10, n. 1, p. 49-63, 2008.

GONÇALVES, A.L.; ALVES FILHO, A.; MENEZES, H. Estudo comparativo da atividade antimicrobiana de extratos de algumas árvores nativas. Arquivo do Instituto Biológico, v. 72, n. 3, p. 353-358, 2005.

GUPTA, R.; THAKUR, B.; SINGH, P.; SINGH, H.B.; SHARMA, V.D.; KATOCH, V.M.; CHAUHAN, S.V.S. Anti-tuberculosis activity of selected medicinal plants against multi-drug resistant Mycobacterium tuberculosis isolates. Indian Journal of Medical Research, v. 131, n. 6, p. 809-813, 2010.

HALAL, I.S.F.; BERTONI, A.M.; CIACOMET, C.; SEIBEL, C.E.; LAHUDE, F.M.; MAGALHÃES, G.A. et al. Avaliação da qualidade de assistência primária à saúde em localidade urbana da região sul do Brasil. Revista

Rev. Bras. PI. Med., Campinas, v.15, n.2, p.188-193, 2013. 
de Saúde Pública, v. 28, n. 2, p. 131-136, 1994.

HENNEBELLE, T.; SAHPAZ, S.; JOSEPH, H.; BAILLEUL,

F. Ethnopharmacology of Lippia alba. Journal of

Ethnopharmacology, v. 116, n. 2, p. $211-212,2008$.

IBGE. Instituto Brasileiro de Geografia e Estatística. Censo 2010. Disponível em http://www.censo2010.ibge.gov.br. Acessado em 10/07/2011. LUCENA, R. F. P.; ALBUQUERQUE, U. P.; MONTEIRO, J. M.; ALMEIDA, C. F. C. B. R.; FLORENTINO, A. T. N.; FERRAZ, J. S. F. Useful plants of the semiarid northeastern region of Brazil - a look at their conservation and sustainable use. Environmental Monitoring and Assessment, v. 125, p. 281-290, 2007.

MARINHO, M.G.V.; SILVA, C.C.; ANDRADE, L.H.C. Levantamento etnobotânico de plantas medicinais em área de caatinga no município de São José de Espinharas, Paraíba, Brasil. Revista Brasileira de Plantas Medicinais, v.13, n.2, p.170-182, 2011.

MARINOFF, M.A.; MARTINEZ, J.L.; URBINA, M.A. Precautions in the use of medicinal. Boletin Latinoamericano y del Caribe de Plantas Medicinales y Aromaticas, v. 8, n. 3, p. 184-187, 2009.

OWOLABI, M.S.; LAJIDE, L.; OLADIMEJI, M.O.; SETZER, W.N.; PALAZZO, M.C. OLOWU, R.A.; OGUNDAJO, A. Volatile Constituents and Antibacterial Screening of the Essential Oil of Chenopodium ambrosioides L. growing in Nigeria. Natural Product Communications, v. 4, n. 7, p. 989-992, 2009.

PEREIRA, E.M.R; GOMES, R.T.; FREIRE, N.R.; AGUIAR, E.G.; BRANDÃO, M.G.L; SANTOS, V.R. In vitro Antimicrobial Activity of Brazilian Medicinal Plant Extracts against Pathogenic Microorganisms of Interest to Dentistry. Planta Medica, v. 77, n. 4, p. 401-404, 2011.

PILLA, M.A.C.; AMOROZO, M.C.M.; FURLAN, A. Obtenção e uso de plantas medicinais no distrito de Martim Francisco, município de Mogi-Mirim, SP, Brasil. Acta Botanica Brasilica, v. 20, n. 4, p. 789-802, 2006. ROSA, C.; CÂMARA, S.G.; BÉRIA, J.U. Representações e intenção de uso da fitoterapia na atenção básica à saúde. Ciências \& Saúde Coletiva, v, 16, n. 1, p. 311
$-318,2011$.

ROSSATO, M.; BARBIERI, R.L. Estudo etnobotânico de palmeiras do Rio Grande do Sul. Revista Brasileira de Agroecologia, v. 2, n. 1, p. 123-127, 2007.

RUELA, H.; LEAL, I.C.R.; ALMEIDA, M.R.A.; SANTOS, K.R.N.; WESSJOHANN, L.A.; KUSTER, R.M. Antibacterial and antioxidant activities and acute toxicity of Bumelia sartorum, a Brazilian medicinal plant. Revista Brasileira de Farmacognosia, v. 21, n. 1, p. 86-91, 2011.

SANTOS, E.B.; DANTAS, G.S.; SANTOS, H.B.; MELO DINIZ, M.F.F.; SAMPAIO, F.C. Estudo etnobotânico de plantas medicinais para problemas bucais no município de João Pessoa, Brasil. Revista Brasileira de Farmacognosia, v. 19, n. 1B, p. 321-324, 2009.

SILVA, M.I.G.; GONDIM, A.P.S.; NUNES, I.F.S.; SOUSA, F.C. Utilização de fitoterápicos nas unidades básicas de atenção à saúde da família no município de Maracanaú (CE). Revista Brasileira de Farmacognosia, v. 16, n. 4, p. 455-462, 2006.

SILVA, D.; MAGALHÃES, V.; MACÊDO, T.; ALMASSY, J.A.; SILVA, F. Levantamento etnofarmacológico em comunidades rurais do Recôncavo da Bahia/BA. Revista Brasileira de Agroecologia, v. 4, n. 2, p. 697-701, 2009.

SILVA, M.A.B.; MELO, L.V.L.; RIBEIRO, R.V.; SOUZA, J.P.M.; LIMA, J.C.S.; MARTINS, D.T.O., et al. Levantamento etnobotânico de plantas utilizadas como anti-hiperlipidêmicas e anorexígenas pela população de Nova Xavantina-MT, Brasil. Revista Brasileira de Farmacognosia, v. 20, n. 4, p. 549-562, 2010.

SILVEIRA, P.F.; BANDEIRA, M.A.M.; ARRAIS, P.S.D. Farmacovigilância e reações adversas às plantas medicinais e fitoterápicos: uma realidade. Revista Brasileira de Farmacognosia, v. 18, n. 4, p. 618-626, 2008.

UCHGAONKAR, P.; DAHIYA, N. Phytochemical Analysis and Antibacterial Activity of Punica granatum L. Rind Extracts on Common Enteric. Journal of Pure and Applied Microbiology, v. 5, n. 1, p. 417-420, 2011. 